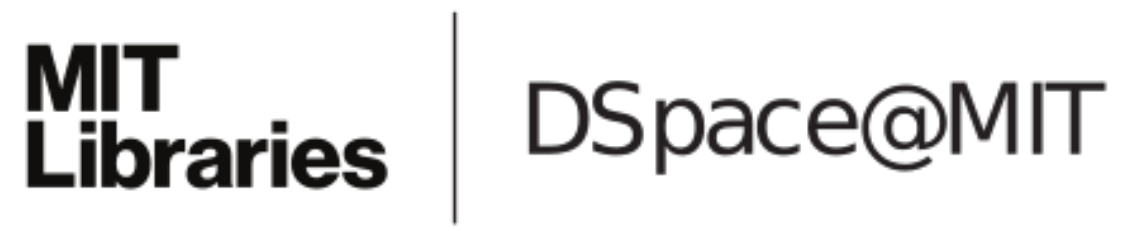

MIT Open Access Articles

Hydrodynamic length-scale selection
in microswimmer suspensions

The MIT Faculty has made this article openly available. Please share how this access benefits you. Your story matters.

Citation: Heidenreich, Sebastian; Dunkel, Jörn; Klapp, Sabine H. L. and Bär, Markus.

"Hydrodynamic length-scale selection in microswimmer suspensions." Physical Review E 94, 020601(R): 1-6 (c) 2016 American Physical Society

As Published: http://dx.doi.org/10.1103/PhysRevE.94.020601

Publisher: American Physical Society

Persistent URL: http://hdl.handle.net/1721.1/110385

Version: Final published version: final published article, as it appeared in a journal, conference proceedings, or other formally published context

Terms of Use: Article is made available in accordance with the publisher's policy and may be subject to US copyright law. Please refer to the publisher's site for terms of use. 


\title{
Hydrodynamic length-scale selection in microswimmer suspensions
}

\author{
Sebastian Heidenreich, ${ }^{1,{ }^{*}}$ Jörn Dunkel, ${ }^{2}$ Sabine H. L. Klapp, ${ }^{3}$ and Markus Bär ${ }^{1}$ \\ ${ }^{1}$ Department of Mathematical Modelling and Data Analysis, Physikalisch-Technische Bundesanstalt Braunschweig und Berlin, \\ Abbestrasse 2-12, D-10587 Berlin, Germany \\ ${ }^{2}$ Department of Mathematics, Massachusetts Institute of Technology, 77 Massachusetts Avenue E17-412, Cambridge, \\ Massachusetts 02139-4307, USA \\ ${ }^{3}$ Institute for Theoretical Physics, Technical University Berlin, Hardenbergstrasse 36, D-10623 Berlin, Germany
} (Received 29 September 2015; revised manuscript received 25 December 2015; published 9 August 2016)

\begin{abstract}
A universal characteristic of mesoscale turbulence in active suspensions is the emergence of a typical vortex length scale, distinctly different from the scale invariance of turbulent high-Reynolds number flows. Collective length-scale selection has been observed in bacterial fluids, endothelial tissue, and active colloids, yet the physical origins of this phenomenon remain elusive. Here, we systematically derive an effective fourth-order field theory from a generic microscopic model that allows us to predict the typical vortex size in microswimmer suspensions. Building on a self-consistent closure condition, the derivation shows that the vortex length scale is determined by the competition between local alignment forces, rotational diffusion, and intermediate-range hydrodynamic interactions. Vortex structures found in simulations of the theory agree with recent measurements in Bacillus subtilis suspensions. Moreover, our approach yields an effective viscosity enhancement (reduction), as reported experimentally for puller (pusher) microorganisms.
\end{abstract}

DOI: 10.1103/PhysRevE.94.020601

A universal feature shared by many living systems is the emergence of characteristic length and time scales that arise from the nonequilibrium dynamics of their microscopic constituents. Examples range from circadian oscillations in individual cells [1] to multicellular gene-expression patterns in embryos [2] and vortex structures in microbial suspensions, endothelial tissue, and active colloids [3-6]. Yet, despite their broad biological relevance, it has proved difficult to predict quantitatively how such emergent scales arise from the underlying chemical or physical parameters. In the past decade, bacterial and other active suspensions $[4,5,7]$ have emerged as important biophysical model systems that can help bridge the gap between large-scale spatiotemporal pattern formation and microscopic nonequilibrium dynamics [8]. At high densities, bacterial fluids form coherent vortex structures, spanning several cell lengths in diameter [5,7,9] and persisting for several seconds [9] or even minutes [10-12]. Although a number of insightful theoretical models have been proposed [13-18], a theory connecting microswimmer properties to the experimentally observed vortex patterns has been lacking.

Here, we present such a theory by drawing guidance from the recent observation [5,9] that an effective fourth-order continuum model can provide a quantitative phenomenological description of dense bacterial suspensions [19]. This model, which combines the seminal Toner-Tu description of flocking [20] with the Swift-Hohenberg equation from pattern formation [21], describes the effective bacterial velocity field $\mathbf{w}(t, \mathbf{x})$ by

$$
\begin{aligned}
\left(\partial_{t}-\lambda_{0} \mathbf{w} \cdot \nabla\right) \mathbf{w}= & -\nabla q+\lambda_{1} \nabla|\mathbf{w}|^{2}+\alpha \mathbf{w}-\beta|\mathbf{w}|^{2} \mathbf{w} \\
& +\Gamma_{0} \nabla^{2} \mathbf{w}-\Gamma_{2}\left(\nabla^{2}\right)^{2} \mathbf{w},
\end{aligned}
$$

where the bacterial pressure field $q(t, \mathbf{x})$ accounts for incompressibility, $\boldsymbol{\nabla} \cdot \mathbf{w}=0$. Although a direct fit of Eq. (1) can

*sebastian.heidenreich@ptb.de reproduce the key statistical features of experimental data for dense quasi-two-dimensional (2D) [5] and three-dimensional (3D) [9] B. subtilis suspensions, the connection between the phenomenological parameters $\left(\lambda_{0}, \lambda_{1}, \alpha, \beta, \Gamma_{0}, \Gamma_{2}\right)$ and individual bacterial properties has remained unknown. Below, we systematically derive a generalized variant of Eq. (1) directly from a generic model for polar microswimmers. The derivation specifies each parameter in the continuum theory in terms of microscopic swimmer parameters and yields direct theoretical predictions for the typical vortex size and effective viscosity in dense microswimmer suspensions. Compared with previous studies, our approach differs in that we deduce a self-consistent closure condition that accounts for shear-induced tumbling and active stress up to fourth order. Both contributions are important to explain the length-scale selection. We present a bifurcation analysis of the resulting fourth-order model and discuss numerical results, demonstrating satisfactory agreement with available experimental data for quasi-2D suspensions [22].

Microscopic model. We consider microswimmers moving in an incompressible Newtonian fluid at low Reynolds number, described by the Stokes equations

$$
-\nabla p+\mu \nabla^{2} \mathbf{u}+\nabla \cdot \boldsymbol{\sigma}=\mathbf{0}, \quad \nabla \cdot \mathbf{u}=0 .
$$

Here, $\mathbf{u}(t, \mathbf{x})$ is the fluid velocity, $p(t, \mathbf{x})$ the hydrodynamic pressure, and $\mu$ the effective dynamic viscosity. The active stress tensor $\sigma$ represents the forcing of the fluid by the swimmers $[8,16,17]$. For dense suspensions, the bulk viscosity $\mu$ contains contributions from the solvent as well as passive and active contributions from the microswimmers [23-28]. For simplicity, we assume that the passive contribution is approximately given by the Batchelor-Einstein relation for spherical colloids, $\mu=\mu_{0}\left(1+k_{1} \phi+k_{3} \phi^{2}\right)$, where $\mu_{0}$ is the "bare" solvent viscosity, $\phi$ is the volume fraction, and $k_{i}$ are positive constants $[23,29-31]$. The active contribution of the linear part will be derived below. In quasi-2D Hele-Shaw flow, 


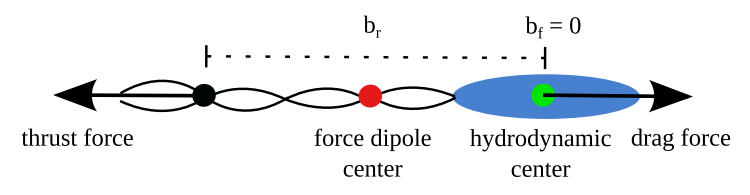

FIG. 1. Schematic of a bacterial microswimmer such as $B$. subtilis. The center of the hydrodynamic stress $\boldsymbol{X}$ (green) is located in front of the force dipole center (red) [33]. $b_{r}$ and $b_{f}$ are the distances between the center $\boldsymbol{X}$ and acting forces. The drag force defines the swimmer orientation $N$.

an effective boundary-friction term $-v_{0} \mathbf{u}$ is added [32] on the left-hand side of the Stokes equation (2).

Focusing on time scales larger than a typical stroke period, we describe microswimmers as force dipoles of strength $f_{0}$ [33] (Fig. 1). Assuming $\sigma=1, \ldots, M$ identical swimmers, their time-dependent positions $\mathbf{X}^{\sigma}(t)$ and orientation unit vectors $\mathbf{N}^{\sigma}(t)$ are determined by the overdamped Langevin equations $[34,35]$

$$
\begin{gathered}
\dot{\mathbf{X}}^{\sigma}=v_{0} \mathbf{N}^{\sigma}+\mathbf{u}\left(t, \mathbf{X}^{\sigma}\right)+\sqrt{2 D} \xi^{\sigma} \\
\dot{\mathbf{N}}^{\sigma}=\boldsymbol{\Pi}^{\sigma} \cdot\left[\nabla \mathbf{u} \cdot \mathbf{N}^{\sigma}-\nabla_{\mathbf{N}^{\sigma}} \Phi+\frac{1}{\sqrt{\tau}} \eta^{\sigma}\right],
\end{gathered}
$$

where overdots indicate time derivatives. The translational dynamics (3) is caused by self-swimming at speed $v_{0}$, hydrodynamic advection $\mathbf{u}\left(t, \mathbf{X}^{\sigma}\right)$, and translational Brownian motion of strength $D$. The random functions $\boldsymbol{\xi}^{\sigma}(t)$ and $\boldsymbol{\eta}^{\sigma}(t)$ denote independent $\delta$-correlated Gaussian white noise. The orientational dynamics (4), interpreted as a Stratonovich stochastic differential equation [36] with rotational relaxation time $\tau$, conserves the length of the orientation vector $\mathbf{N}^{\sigma}$ by virtue of the projector $\Pi^{\sigma}=\mathbf{I}-\mathbf{N}^{\sigma} \mathbf{N}^{\sigma}$, where $\mathbf{I}$ is the unit matrix. The $\nabla \mathbf{u}$ term accounts for reorientation of elongated particles by flow gradients in the limit of large aspect ratios [37,38]. In dense suspensions of fast-swimming bacteria, steric collisions are negligible in the translation dynamics but may contribute significantly to reorientation. We therefore include a polar reorientation interaction potential $\Phi\left(\mathbf{N}^{\sigma}\right)=$ $-g \sum_{\left|\mathbf{X}^{\sigma}-\mathbf{X}^{v}\right| \leqslant \epsilon} \mathbf{N}^{\sigma} \cdot \mathbf{N}^{v}$ with a cutoff length $\epsilon$ and alignment strength $g=g_{0} v_{0} / 2$ in Eq. (4), reflecting the experimental observation of locally aligned bacterial jets $[5,6,39]$. In particular, we assume that kinematically induced polar interactions dominate over nematic ordering [40], the latter representing the dominant alignment force in the passive limit $v_{0} \rightarrow 0$. It is worth emphasizing that the assumed dominance of effectively polar alignment interactions is essential and distinguishes our model from previous studies of active nematics $[15,41]$ and nematically interacting microswimmers [35].

Although Eqs. (3) and (4) as well as the main steps of the subsequent derivation remain valid for $3 \mathrm{D}$ bulk suspensions, we focus, for clarity, on free-standing quasi-2D films $[22,42,43]$ from now on.

Fokker-Planck dynamics. To derive a continuum model from Eqs. (2)-(4), we consider the one-particle distribution $\mathcal{P}(t, \mathbf{x}, \mathbf{n})=M^{-1} \sum_{\sigma=1}^{M}\left\langle\delta\left(\mathbf{x}-\mathbf{X}^{\sigma}\right) \delta\left(\mathbf{n}-\mathbf{N}^{\sigma}\right)\right\rangle$, where $\langle\cdot\rangle$ denotes an average over the Gaussian white noise $\left\{\boldsymbol{\xi}^{\sigma}(t), \boldsymbol{\eta}^{\sigma}(t)\right\}$. The evolution of $\mathcal{P}(t, \mathbf{x}, \mathbf{n})$ is governed by the Fokker-Planck equation [36,44] (see the Supplemental Material [45])

$$
\begin{aligned}
\partial_{t} \mathcal{P}= & -\nabla \cdot\left(v_{0} \mathbf{n}+\mathbf{u}\right) \mathcal{P}+D \nabla^{2} \mathcal{P}-\nabla_{\mathbf{n}} \cdot \boldsymbol{\Pi} \cdot(\nabla \mathbf{u}) \cdot \mathbf{n} \mathcal{P} \\
& +\frac{1}{\tau} \nabla_{\mathbf{n}} \cdot \mathbf{n} \mathcal{P}+\frac{1}{2 \tau} \nabla_{\mathbf{n}} \nabla_{\mathbf{n}}:\left(\boldsymbol{\Pi} \cdot \boldsymbol{\Pi}^{T}\right) \mathcal{P}+\mathcal{C}^{(2)}[\Phi]
\end{aligned}
$$

Alignment interactions enter via the collision integral

$$
\mathcal{C}^{(2)}[\Phi]=\nabla_{\mathbf{n}} \cdot \int d \mathbf{n}^{\prime} \int_{\epsilon} d \mathbf{x}^{\prime} \boldsymbol{\Pi} \cdot\left[\nabla_{\mathbf{n}} \Phi\left(\mathbf{n}, \mathbf{n}^{\prime}\right)\right] \mathcal{P}^{(2)},
$$

which involves the two-particle distribution function $\mathcal{P}^{(2)}\left(t, \mathbf{x}, \mathbf{n} ; \mathbf{x}^{\prime}, \mathbf{n}^{\prime}\right)$.

Moment equations. To derive hydrodynamic field equations from Eqs. (5) and (6), we define the 2D swimmer number density $\rho(t, \mathbf{x})=M \int d \mathbf{n} \mathcal{P}$, the polar order-parameter field $\mathbf{P}(t, \mathbf{x})=\overline{\mathbf{n}}$, and the nematic order-parameter field $\mathbf{Q}(t, \mathbf{x})=$ $(\overline{\mathbf{n n}}-\mathbf{I} / 2)$, where the bar denotes the marginal average over all orientations $\overline{\mathbf{g}}(t, \mathbf{x})=\int d \mathbf{n} \mathbf{g}(\mathbf{n}) \mathcal{P} / \int d \mathbf{n} \mathcal{P}$. Integrating Eq. (5) over $\mathbf{n}$, considering the limit of constant density $\rho$, and using $\boldsymbol{\nabla} \cdot \mathbf{u}=0$ implies incompressibility of the orientation field, $\boldsymbol{\nabla} \cdot \mathbf{P}=0$ (see the Supplemental Material [45]).

To obtain the dynamic equation for $\mathbf{P}$, we multiply Eq. (5) by $\mathbf{n}$ and integrate over all orientations. Adopting a standard mean-field approximation for Eq. (6), assuming again constant density $\rho$, and neglecting terms of tensorial rank higher than two, we find (see the Supplemental Material [45])

$$
\begin{aligned}
\partial_{t} \mathbf{P}+\mathbf{u} \cdot \nabla \mathbf{P}= & -\nabla \hat{p}-v_{0} \nabla \cdot \mathbf{Q} \\
& +\left(D+D_{\epsilon}\right) \nabla^{2} \mathbf{P}+\left(\epsilon^{2} / 24\right) D_{\epsilon} \nabla^{4} \mathbf{P} \\
& +\left[\Omega+(1 / 2) \mathbf{P} \cdot \Sigma+\left(4 / \epsilon^{2}\right) D_{\epsilon}(\mathbf{I}-2 \mathbf{Q})\right. \\
& -(1 / \tau) \mathbf{I}] \cdot \mathbf{P}
\end{aligned}
$$

where $\nabla^{4}=\left(\nabla^{2}\right)^{2}$. The local Lagrange multiplier $\hat{p}(t, \mathbf{x})$ ensures a divergence-free orientation field, $\boldsymbol{\Sigma}=\frac{1}{2}[\nabla \mathbf{u}+$ $\left.(\nabla \mathbf{u})^{\top}\right]$ is the hydrodynamic rate-of-strain tensor, $\boldsymbol{\Omega}$ is the antisymmetric part of $\nabla \mathbf{u}$, and $D_{\epsilon}=\rho g_{0} v_{0} \pi \epsilon^{4} / 8$ encodes translational diffusion caused by mean-field polar interactions. The destabilizing $\left(\epsilon^{2} / 24\right) D_{\epsilon} \nabla^{4} \mathbf{P}$ term, arising from polar interactions, is counteracted by the $\nabla \cdot \mathbf{Q}$ term.

To connect with Eq. (1), we introduce an nonconserved self-swimming velocity field $\mathbf{v}(t, \mathbf{x})=v_{0} \mathbf{P}$. The total velocity field of the microswimmers, appearing in Eq. (1) and measured in experiments [5,7,9], is given by $\mathbf{w}=\mathbf{u}+\mathbf{v}$, corresponding to the hydrodynamic average of Eq. (3).

Self-consistent closure. To close Eq. (7), we have to approximate the nematic order-tensor $\mathbf{Q}$ in terms of $\mathbf{P}$ and u. A simple closure condition for passive hard rods [46] is $\mathbf{Q} \sim(\mathbf{P P})^{+}$, where $\mathbf{A}^{+}$with components $A_{i j}^{+}=\left(A_{i j}+A_{j i}-\right.$ $\left.\delta_{i j} A_{k k}\right) / 2$ denotes the symmetric traceless part of tensor $\mathbf{A}$ in 2D. However, this commonly used closure condition does not account for the fact that active microswimmers permanently impose stress on the ambient fluid which feeds back into the orientational order, analogous to the shear-induced isotropicnematic transition in liquid crystals [47-50]. To derive a selfconsistent closure condition, we multiply Eq. (5) by $\mathbf{n n}$ and then integrate over all orientations. Taking the stationary limit of the resulting equation, assuming small spatial variations of $\mathbf{Q}$, and neglecting "flexoelectric" contributions [51], one finds

$$
\mathbf{Q}=\lambda_{\mathrm{P}}(\mathbf{P P})^{+}+\lambda_{\Sigma} \mathbf{\Sigma},
$$


with $\lambda_{\mathrm{P}}=\left(2 / \epsilon^{2}\right) D_{\epsilon} \tau$ and tumbling parameter $\lambda_{\Sigma}=\tau / 16$.

Hydrodynamic stress. As a last step, we have to relate the active stress tensor $\boldsymbol{\sigma}$ in Eq. (2) to P. To this end, note that the swimmer position $\mathbf{X}^{\sigma}$ coincides with the center of hydrodynamic stress (Fig. 1), which is the point where the hydrodynamic net torque on a rigid body vanishes [52]. For an asymmetric dipole swimmer of effective length $\ell=b_{f}+b_{r}$ with $b_{f}, b_{r}>0$, the propulsive rear force $\mathbf{F}_{r}^{\sigma}=-f_{0} \mathbf{N}^{\sigma}$ acts at $\mathbf{X}_{r}^{\sigma}=\mathbf{X}^{\sigma}-b_{r} \mathbf{N}^{\sigma}$, and the resistive front force $\mathbf{F}_{f}^{\sigma}=f_{0} \mathbf{N}^{\sigma}$ at $\mathbf{X}_{f}^{\sigma}=\mathbf{X}^{\sigma}+b_{f} \mathbf{N}^{\sigma}$ (Fig. 1). The swimmer is force free, $\mathbf{F}_{r}^{\sigma}+\mathbf{F}_{f}^{\sigma}=\mathbf{0}$, and for bacterial pusher dipoles we have $f_{0}>0$ [33]. The total force density is then given by

$$
\mathbf{f}=\nabla \cdot \boldsymbol{\sigma}=\sum_{\sigma} \mathbf{F}_{r}^{\sigma} \delta\left(\mathbf{x}-\mathbf{X}_{r}^{\sigma}\right)+\mathbf{F}_{f}^{\sigma} \delta\left(\mathbf{x}-\mathbf{X}_{f}^{\sigma}\right) .
$$

Taylor-expanding for small $b_{r}$ and $b_{f}$, assuming constant density across a thin film of thickness $h \sim \ell$, neglecting terms of order $(\nabla \mathbf{P})^{2}$, and averaging over noise and orientations $[16,17]$, we find for the divergence of the averaged total stress tensor [53]

$$
\boldsymbol{\nabla} \cdot \overline{\boldsymbol{\sigma}}=-\frac{f_{0} \rho}{h} \nabla \cdot\left[\ell \mathbf{Q}+2\left(\gamma_{2}+\gamma_{4} \nabla^{2}\right)(\boldsymbol{\nabla} \mathbf{P})^{+}\right],
$$

with $\gamma_{2}=\left(b_{r}^{2}-b_{f}^{2}\right) / 8>0$ and $\gamma_{4}=\left(b_{r}^{4}-b_{f}^{4}\right) / 192>0$ for pushers (in $3 \mathrm{D}, \rho / h$ is replaced by the concentration $c$ ). The divergence of the symmetric traceless gradient tensor $(\nabla \mathbf{P})^{+}$ is proportional to the Laplacian of the orientation field $\mathbf{P}$. Combining Eqs. (8) and (10) with (2) and (7), we obtain a closed set of equations for the two incompressible vector fields $(\mathbf{u}, \mathbf{P})$ and their associated pressure fields.

Stokes equations and viscosity. Inserting Eq. (10) into Eq. (2), the Stokes equation can be written as

$$
\mu_{*} \nabla^{2} \mathbf{u}-\nabla p_{*}=f_{0} \rho\left(\ell \lambda_{\mathrm{P}} \mathbf{P} \cdot \nabla+\gamma_{2} \nabla^{2}+\gamma_{4} \nabla^{4}\right) \mathbf{P},
$$

with effective viscosity and pressure given by

$$
\begin{aligned}
& \mu_{*}=\mu-\frac{\ell \lambda_{\Sigma}}{2} \frac{f_{0} \rho}{h}, \\
& p_{*}=p-\frac{\ell \lambda_{\mathrm{P}}}{2}|\mathbf{P}|^{2} \frac{f_{0} \rho}{h} .
\end{aligned}
$$

Rewriting $\mu_{*}$ in terms of the volume fraction $\phi=\rho A$, where $A$ is the projected 2D area of a swimmer, and choosing $h=\ell$, we find

$$
\mu_{*}=\mu_{0}\left[1+\left(k_{1}-k_{2}\right) \phi+k_{3} \phi^{2}\right],
$$

where $k_{1}=5 / 2$ for passive spherical objects [23,29-31] and $k_{2}=f_{0} \lambda_{\Sigma}\left(2 \mu_{0} A\right)^{-1}$. Thus, our theory implies that, to linear order in $\phi$, pushers with $f_{0}>0$ can reduce the viscosity whereas pullers with $f_{0}<0$ generally enhance the viscosity. In $3 \mathrm{D}$, an analogous derivation yields $k_{2}=f_{0} \ell \lambda_{\Sigma}\left(2 \mu_{0} V_{b}\right)^{-1}$, where $V_{b}$ is the effective volume of the individual swimmer. For pullers, the coefficient $k_{3}>0$ is chosen according to Refs. [23,29], and for pushers we determine $k_{3}>0$ directly from the data (Fig. 2). Comparing Eq. (13) against experimental data yields good agreement with recent measurements in 3D bacterial [24,25] and algal [26] suspensions (Fig. 2). We note that qualitatively similar results for the effective viscosity were obtained recently for other basic microswimmers models [23,54]. (a) Chlamydomonas Reinhardtii

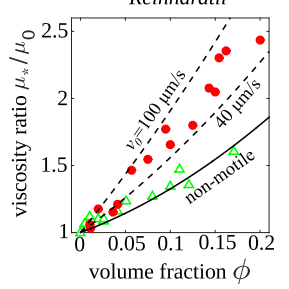

(b) Escherichia coli

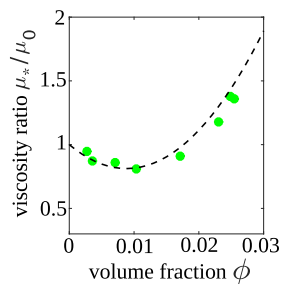

(c) Bacillus subtilis

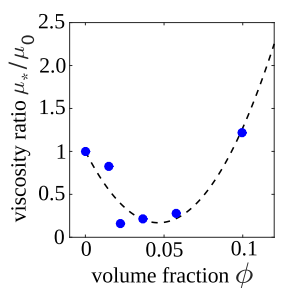

FIG. 2. Comparison of predictions (dashed lines) based on Eq. (13) with recent experiments (symbols) for force density values $f_{0}$ given in the text. (a) For Chlamydomonas algae (puller), the theory predicts an increase in the viscosity for motile cells with $k_{2}>0$ (dashed lines, using $\ell=5 \mu \mathrm{m}, \tau=3.5 \mathrm{~s}, V_{b}=294.5 \mu \mathrm{m}^{3}$, $k_{3}=7.6[23,29]$ ) compared with nonmotile cells (solid line, $k_{2}=0$ ), in agreement with recent measurements for motile (red circles) and dead (green triangles) cells [26]. (b), (c) For bacteria (pusher) with $k_{2}<0$, the theory correctly reproduces a viscosity decrease at intermediate filling fractions. Dashed curves are based on the following fit parameters: (b) For E. coli data (green circles [25]): $v_{0}=20 \mu \mathrm{m} / \mathrm{s}, V_{b}=1.57 \mu \mathrm{m}^{3}, k_{3}=2400, \tau=4.8 \mathrm{~s}$. (c) For $B$. subtilis data (blue circles [24]): $v_{0}=30 \mu \mathrm{m} / \mathrm{s}, V_{b}=1.92 \mu \mathrm{m}^{3}$, $k_{3}=385, \tau=0.5 \mathrm{~s}$.

Orientation field dynamics. Taking the divergence of Eq. (8) and utilizing the Stokes equation (11) yields $\boldsymbol{\nabla} \cdot \mathbf{Q}$ in terms of the orientation field $\mathbf{P}$. Substituting the resulting expression into Eq. (7), we find the fourth-order partial differential equation (PDE)

$$
\begin{aligned}
\partial_{t} \mathbf{P} & +\left(\mathbf{u}+v_{0} \lambda_{\mathrm{P} *} \mathbf{P}\right) \cdot \nabla \mathbf{P} \\
= & -\nabla \hat{p}_{*}+\mathbf{\Omega} \cdot \mathbf{P}+\left[1 / 2-\left(8 / \epsilon^{2}\right) D_{\epsilon} \lambda_{\Sigma}\right] \mathbf{\Sigma} \cdot \mathbf{P} \\
& +\alpha \mathbf{P}-\beta|\mathbf{P}|^{2} \mathbf{P}+\Gamma_{0} \nabla^{2} \mathbf{P}-\Gamma_{2} \nabla^{4} \mathbf{P},
\end{aligned}
$$

where $\lambda_{\mathrm{P} *}=\left(\mu / \mu_{*}\right) \lambda_{\mathrm{P}}$ and the effective orientation-pressure field is given by

$$
\hat{p}_{*}=\hat{p}+v_{0}\left(-\frac{\lambda_{\mathrm{P}}}{2}|\mathbf{P}|^{2}+\frac{\lambda_{\Sigma}}{2 \mu_{*}} p_{*}\right) .
$$

The remaining parameters in Eq. (14) are obtained as

$$
\begin{aligned}
\Gamma_{0} & =\left(D+D_{\epsilon}\right)-\frac{\lambda_{\Sigma} v_{0} f_{0} \phi}{2 \mu_{*} A \ell} \gamma_{2}, \\
\alpha & =\frac{4 D_{\epsilon}}{\epsilon^{2}}-\frac{1}{\tau}, \\
\Gamma_{2} & =\frac{\lambda_{\Sigma} v_{0} f_{0} \phi}{2 \mu_{*} A \ell} \gamma_{4}-\frac{\epsilon^{2} D_{\epsilon}}{24}, \\
\beta & =\frac{4 D_{\epsilon}}{\epsilon^{2}} \lambda_{\mathrm{P}} .
\end{aligned}
$$

For clarity, we summarize parameters and relations between them in Table I.

Equation (14) is structurally similar to the Toner-Tu equation [20] with the significant difference that the "diffusion" parameter $\Gamma_{0}$ can become negative when the volume fraction $\phi$ and active power $v_{0} f_{0}$ become sufficiently large, as proposed earlier on purely phenomenological grounds [5,9,19]. For $\Gamma_{0}<0$, Eq. (14) predicts a transition to mesoscale turbulence, as observed in dense $B$. subtilis suspensions [5,7,9]. 
TABLE I. Summary of key parameters and typical values for B. subtilis suspensions used in our simulations, based on Refs. [5,7,9,24,55].

\begin{tabular}{lcc}
\hline \hline & Value & Description \\
\hline$\ell$ & $5.0 \mu \mathrm{m}$ & Effective dipole length \\
$d$ & $0.7 \mu \mathrm{m}$ & Width of the swimmer \\
$v_{0}$ & $1 \ldots 40 \mu \mathrm{m} / \mathrm{s}$ & Self-propulsion speed \\
$\phi$ & 0.4 & Volume fraction \\
$\tau$ & $0.01 \ldots 10^{4} \mathrm{~s}$ & Rotational noise \\
$g_{0}$ & $0.001 \ldots 10^{3} \mu \mathrm{m}^{-1}$ & Strength of polar alignment \\
$\epsilon$ & $3.0 \mu \mathrm{m}$ & Polar alignment range \\
$D$ & $0 \ldots 5 \mu \mathrm{m}$ & Diffusion length \\
$D_{\epsilon}$ & $\rho g_{0} v_{0} \pi \epsilon^{4} / 8$ & Alignment diffusion \\
$\lambda_{\Sigma}$ & $\tau / 16$ & Tumbling parameter \\
$f_{0}$ & $2 \pi \mu_{0} \ell v_{0}$ & Force density \\
$\mu_{*}$ & $\mu_{0}\left[1-\left(k_{1}-k_{2}\right) \phi+k_{3} \phi\right]$ & Effective bulk viscosity \\
$\lambda_{P}$ & $2 D_{\epsilon} \tau \epsilon^{-2}$ & Advection strength \\
$\gamma_{2}$ & $\ell^{2} / 8$ & HD stress expansion coeff. \\
$\gamma_{4}$ & $\ell^{4} / 192$ & HD stress expansion coeff. \\
$A$ & $\ell d$ & Projected 2D area \\
$\lambda_{\mathrm{P} *}$ & $\mu \mu_{*}^{-1} \lambda_{\mathrm{P}}$ & Scaled advection strength \\
\hline \hline
\end{tabular}

Parameters. The coefficients in Eqs. (11)-(16) can be directly estimated from experiments [5,7,9,24,55]: In our simulations, we consider parameters for $B$. subtilis bacteria (cell length $\sim 5 \mu \mathrm{m}$ and diameter $d=0.7 \mu \mathrm{m}$ ) at high volume fractions $\phi \sim 0.4$ [5,55], assuming an effective dipole length $\ell=b_{r}=5 \mu \mathrm{m}$ (Fig. 1) and for the projected 2D area $A \approx d \ell$. The typical force $f_{0}$ exerted by a single microswimmer on the surrounding fluid can be estimated as $f_{0} \approx 2 \pi \mu_{0} \ell v_{0}$ [55], with a typical bacterial self-propulsion speed $v_{0} \in[1,50] \mu \mathrm{m} / \mathrm{s}$ [22]. In the collision-dominated highdensity regime relevant to our study, translational Brownian motion is negligible, $D \ll D_{\epsilon}$, and we set $D=0$ in our simulations. We further assume that steric short-range interactions occur predominantly on the length scale of the cell body, $\epsilon=3 \mu \mathrm{m}$. After fixing the above parameter values, we can analyze how changes of the rotational relaxation time $\tau$, alignment strength $g_{0}$, and swimming activity $v_{0}$ affect the collective dynamics, by exploring the range $\tau \sim 0.01 \ldots 10^{4} \mathrm{~s}$, $g_{0}=0.001 \ldots 10^{3} \mu \mathrm{m}^{-1}$, and $v_{0}=1 \ldots 40 \mu \mathrm{m} / \mathrm{s}$ (Fig. 3). The typical coefficient values used to model the collective dynamics of B. subtilis are summarized in Table I.

Bifurcation diagram. The field equations (11) and (14) have two fixed points: the disordered state $(\mathbf{u}=\mathbf{0}, \mathbf{P}=\mathbf{0})$ and the polar ordered state $(\mathbf{u}=\mathbf{0}, \mathbf{P} \neq \mathbf{0})$. Upon varying $\tau$ and $g_{0}$, these homogeneous states become unstable when the alignment strength becomes subcritical relative to the rotational noise [red line in Fig. 3(a)]. Conversely, strong alignment stabilizes the homogeneous polar state. Defining $\tau$ as the control parameter and the collective velocity $v_{c}=$ $v_{0} \sqrt{\alpha / \beta}$ as an order parameter, the dotted black line of the state diagram yields the bifurcation diagram of Fig. 3(b). Upon linearizing Eq. (14) about the isotropic state, the typical vortex length follows from the most unstable mode, which has a wavelength $\Lambda \sim 2 \pi \sqrt{2 \Gamma_{2} /\left(-\Gamma_{0}\right)}$. As is evident from the explicit expressions for $\Gamma_{0}, \Gamma_{2}$, and $\alpha$ in Eq. (16), this vortex scale is set by the competition between hydrodynamic
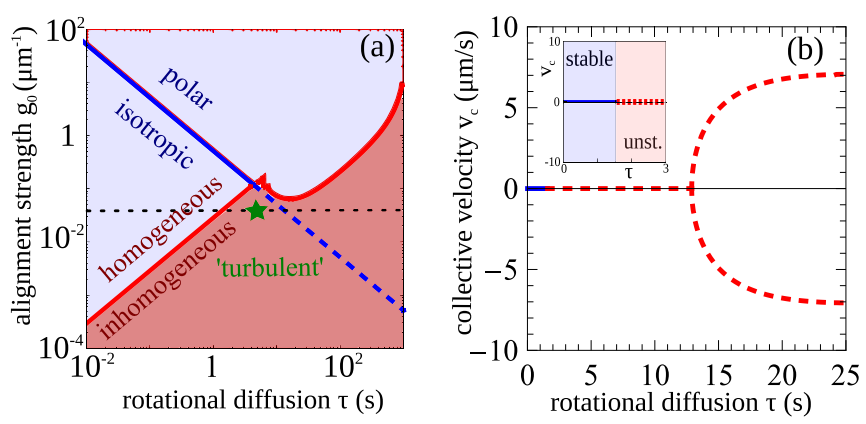

FIG. 3. Bifurcation analysis. (a) State diagram for rodlike pusher obtained by a linear stability analysis of Eq. (14) for typical B. subtilis parameters (see text) and $v_{0}=10 \mu \mathrm{m} / \mathrm{s}$. The red line demarcates the transition to mesoscale turbulence. The blue line signals the transition between disorder and polar order. The purple star indicates the parameters used in simulations. (b) Bifurcation diagram of the collective velocity $v_{c}=v_{0} \sqrt{\alpha / \beta}$ for rodlike pushers along the dotted black line $\left(g_{0}=0.04 \mu \mathrm{m}^{-1}\right)$ in (a). Red dashed lines depict unstable branches, whereas blue solid lines depict stable branches. Inset: Zoom to $\tau \in[0,3] \mathrm{s}$.

flows, steric alignment interactions, activity, and rotational noise. In particular, in the limit of strong self-propulsion and high concentrations, the theoretically predicted vortex size approaches a constant value in agreement with recent experiments [5,7].

Simulations versus experiment. To study the full nonlinear behavior, we solved Eqs. (11) and (14) numerically with a pseudospectral code that combines antialiasing with an operator splitting technique [5]. Simulations were performed using $128 \times 128$ grid points for an area of $101 \times 101 \mu \mathrm{m}^{2}$ and time steps of $d t=10^{-3} \mathrm{~s}$, respectively, for a total simulation time in the range $[500,1000] \mathrm{s}$. For typical $B$. subtilis parameters and $\tau=4.5 \mathrm{~s}, g_{0}=0.04 \mu \mathrm{m}^{-1}$, we obtain flow structures that agree with recently measured flow fields [Fig. 4(a)]. The simulations of the full nonlinear equations
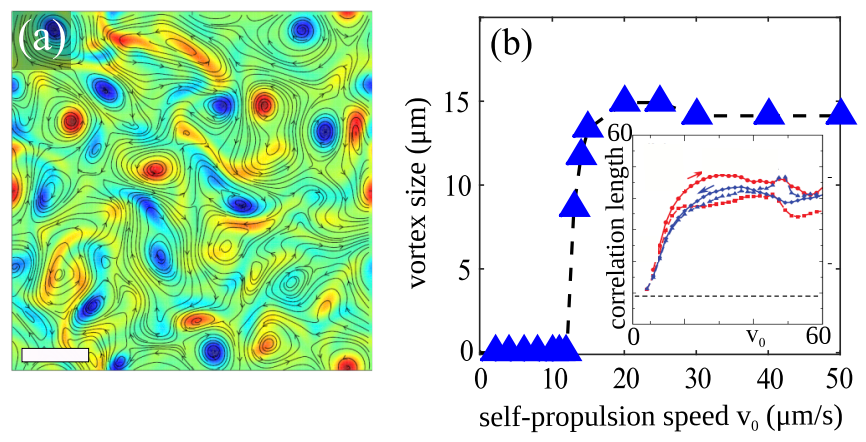

FIG. 4. (a) Representative snapshot of the effective velocity field $\mathbf{w}$ from a simulation with typical $B$. subtilis parameters (see text), $v_{0}=20 \mu \mathrm{m} / \mathrm{s}$ and $g_{0}=0.04 \mu \mathrm{m}^{-1}$. Scale bar corresponds to $20 \mu \mathrm{m}$ and color coding indicates vorticity normalized by the maximum. (b) In dense suspensions, the characteristic vortex size approaches a constant value at large activity. This prediction agrees qualitatively with recent measurements [7] in 3D (inset) (reproduced with permission) and 2D bacterial suspensions [22], which report a typical velocity correlation length of $\sim 20 \mu \mathrm{m}$ (cf. Fig. 5 in Ref. [22]). 
show dynamical vortex structures of a characteristic finite size independent of box size. Moreover, the numerically measured vortex scale, obtained from the minimum of the velocity correlation function, approaches a constant value at large activity, in agreement with recent measurements [7,22] [Fig. 4(b)].

Conclusions. We presented a systematic derivation of higher-than-second-order hydrodynamic equations from a generic microswimmer model. The resulting field theory explains simultaneously a number of recent experimental observations, including the reduction (enhancement) of viscosity in pusher (puller) suspensions and the emergence of a finite characteristic vortex size in dense active fluids from the interplay of rotational diffusion, fluid-dynamical, and polar alignment interactions. Generally, this work shows that higher-order theories of active suspensions [5,9] arise naturally if one adopts self-consistent closure conditions. The relations between microscopic and macroscopic parameters derived here provide a first step towards the quantitative description of bacterial turbulence of $B$. subtilis. Additional improvements will require more accurate microswimmer models and a better understanding of pair correlations. Future efforts may focus on generalizing the above approach to active nematics [56].

Acknowledgments. We are grateful to L. Schimansky-Geier, R. Großmann, P. Romanczuk, I. Aranson, J. Casademunt, and C. Marchetti for discussions. This work was supported by the Deutsche Forschungsgemeinschaft through GRK 1558 and SFB 910 (S.H., S.H.L.K., M.B.), by an MIT Solomon Buchsbaum Fund Award (J.D.), and an Alfred P. Sloan Research Fellowship (J.D.).
[1] J. C. Dunlap, Cell 96, 271 (1999).

[2] S. F. Gilbert, Developmental Biology, 8th ed. (Sinauer Associates, Sunderland, MA, 2006).

[3] D. Nishiguchi and M. Sano, Phys. Rev. E 92, 052309 (2015).

[4] N. S. Rossen, J. M. Tarp, J. Mathiesen, M. H. Jensen, and L. B. Oddershede, Nat. Commun. 5, 5720 (2014).

[5] H. H. Wensink, J. Dunkel, S. Heidenreich, K. Drescher, R. E. Goldstein, H. Löwen, and J. M. Yeomans, Proc. Natl. Acad. Sci. USA 109, 14308 (2012).

[6] H. Zhang, A. Be'er, R. S. Smith, E.-L. Florin, and H. L. Swinney, Europhys. Lett. 87, 48011 (2009).

[7] A. Sokolov and I. S. Aranson, Phys. Rev. Lett. 109, 248109 (2012).

[8] M. Marchetti, J. Joanny, S. Ramaswamy, T. Liverpool, J. Prost, M. Rao, and R. A. Simha, Rev. Mod. Phys. 85, 1143 (2013).

[9] J. Dunkel, S. Heidenreich, K. Drescher, H. H. Wensink, M. Bär, and R. E. Goldstein, Phys. Rev. Lett. 110, 228102 (2013).

[10] H. Wioland, F. G. Woodhouse, J. Dunkel, J. O. Kessler, and R. E. Goldstein, Phys. Rev. Lett. 110, 268102 (2013).

[11] E. Lushi, H. Wioland, and R. E. Goldstein, Proc. Natl. Acad. Sci. USA 111, 9733 (2014).

[12] R. Großmann, P. Romanczuk, M. Bär, and L. Schimansky-Geier, Phys. Rev. Lett. 113, 258104 (2014).

[13] A. Baskaran and M. C. Marchetti, Phys. Rev. E 77, 011920 (2008).

[14] A. Peshkov, I. S. Aranson, E. Bertin, H. Chaté, and F. Ginelli, Phys. Rev. Lett. 109, 268701 (2012).

[15] A. Ahmadi, T. B. Liverpool, and M. C. Marchetti, Phys. Rev. E 72, 060901 (2005).

[16] R. A. Simha and S. Ramaswamy, Phys. Rev. Lett. 89, 058101 (2002).

[17] A. Baskaran and M. C. Marchetti, Proc. Natl. Acad. Sci. USA 106, 15567 (2009).

[18] I. S. Aranson, A. Sokolov, J. O. Kessler, and R. E. Goldstein, Phys. Rev. E 75, 040901(R) (2007).

[19] J. Dunkel, S. Heidenreich, M. Bär, and R. E. Goldstein, New J. Phys. 15, 045016 (2013).

[20] J. Toner and Y. Tu, Phys. Rev. E 58, 4828 (1998).

[21] J. Swift and P. C. Hohenberg, Phys. Rev. A 15, 319 (1977).

[22] A. Sokolov, I. S. Aranson, J. O. Kessler, and R. E. Goldstein, Phys. Rev. Lett. 98, 158102 (2007).
[23] B. M. Haines, I. S. Aranson, L. Berlyand, and D. A. Karpeev, Physical Biology 5, 046003 (2008).

[24] A. Sokolov and I. S. Aranson, Phys. Rev. Lett. 103, 148101 (2009).

[25] J. Gachelin, G. Miño, H. Berthet, A. Lindner, A. Rousselet, and E. Clément, Phys. Rev. Lett. 110, 268103 (2013).

[26] S. Rafai, L. Jibuti, and P. Peyla, Phys. Rev. Lett. 104, 098102 (2010).

[27] S. D. Ryan, B. M. Haines, L. Berlyand, F. Ziebert, and I. S. Aranson, Phys. Rev. E 83, 050904(R) (2011).

[28] S. Gluzman, D. A. Karpeev, and L. V. Berlyand, J. R. Soc., Interface 10, 20130720 (2013).

[29] J. Hinch, J. Fluid Mech. 663, 8 (2010).

[30] G. Batchelor, Annu. Rev. Fluid. Mech. 6, 227 (1974).

[31] A. Einstein, Ann. Phys. 324, 289 (1906).

[32] F. G. Woodhouse and R. E. Goldstein, Proc. Natl. Acad. Sci. USA 110, 14132 (2013).

[33] K. Drescher, J. Dunkel, L. H. Cisneros, S. Ganguly, and R. E. Goldstein, Proc. Natl. Acad. Sci. USA 108, 10940 (2011).

[34] D. Saintillan and M. J. Shelley, Phys. Rev. Lett. 100, 178103 (2008).

[35] D. Saintillan and M. J. Shelley, C. R. Phys. 14, 497 (2013).

[36] K. Jacobs, Stochastic Processes for Physicists: Understanding Noisy Systems (Cambridge University Press, Cambridge, U.K., 2010).

[37] G. B. Jeffery, Proc. R. Soc. London, Ser. A 102, 161 (1922).

[38] C. Hohenegger and M. J. Shelley, in New Trends in the Physics and Mechanics of Biological Systems, Lecture Notes of the Les Houches Summer School, Vol. 92 (Oxford University Press, New York, 2011), pp. 65-93.

[39] H.-P. Zhang, A. Be'er, E.-L. Florin, and H. L. Swinney, Proc. Natl. Acad. Sci. USA 107, 13626 (2010).

[40] F. Peruani, A. Deutsch, and M. Bär, Phys. Rev. E 74, 030904 (2006).

[41] S. P. Thampi, R. Golestanian, and J. M. Yeomans, Phys. Rev. Lett. 111, 118101 (2013).

[42] J. S. Guasto, K. A. Johnson, and J. P. Gollub, Phys. Rev. Lett. 105, 168102 (2010).

[43] H. Kurtuldu, J. S. Guasto, K. A. Johnson, and J. P. Gollub, Proc. Natl. Acad. Sci. USA 108, 10391 (2011).

[44] H. Risken, Fokker-Planck Equation (Springer, Berlin, 1984). 
[45] See Supplemental Material at http://link.aps.org/supplemental/ 10.1103/PhysRevE.94.020601 for information on the derivation of the continuum equations.

[46] M. Doi and S. F. Edwards, The Theory of Polymer Dynamics, Vol. 73 (Oxford University Press, Oxford, U.K., 1988).

[47] S. Hess, Z. Naturforsch., A 30, 1224 (1975).

[48] C. Pujolle-Robic and L. Noirez, Nature (London) 409, 167 (2001).

[49] M. R. López-González, W. M. Holmes, P. T. Callaghan, and P. J. Photinos, Phys. Rev. Lett. 93, 268302 (2004).

[50] P. T. Mather, A. Romo-Uribe, C. D. Han, and S. S. Kim, Macromolecules 30, 7977 (1997).
[51] S. Heidenreich, S. Hess, and S. H. L. Klapp, Phys. Rev. Lett. 102, 028301 (2009).

[52] J. Happel and H. Brenner, Low Reynolds Number Hydrodynamics, International Series in the Physical and Chemical Engineering Sciences (Prentice-Hall, Englewood Cliffs, NJ, 1965).

[53] To simplify subsequent equations, we drop $\mathcal{O}\left(b_{r / f}^{3}\right)$ terms but kept the $\gamma_{4}$ term to ensure stability.

[54] M. Moradi and A. Najafi, Europhys. Lett. 109, 24001 (2015).

[55] C. W. Wolgemuth, Biophys. J. 95, 1564 (2008).

[56] T. Sanchez, D. T. N. Chen, S. J. DeCamp, M. Heymann, and Z. Dogic, Nature (London) 491, 431 (2012). 